\title{
EDITORIAL The challenges of estimating the cost of traumatic brain injury worldwide
}

\author{
Shelly D. Timmons, MD, PhD \\ Department of Neurosurgery, Penn State University Milton S. Hershey Medical Center, Hershey, Pennsylvania
}

$\mathrm{I}$ $\mathrm{N}$ their investigation entitled "The Estimated Cost of Surgically Managed Isolated Traumatic Head Injury Secondary to Road Traffic Accidents,"5 X. You et al. have highlighted many of the challenges inherent in estimating costs of care in general. This group studied the direct costs of traumatic brain injury (TBI) over a finite period in a very well circumscribed group of TBI patients in what was admittedly only a beginning effort to understand costs of care and the societal financial impact of TBI in Malaysia. Given the authors' citation that $7.54 \%$ of hospital admissions to Ministry of Health and private hospitals in Malaysia in 2015 were due to injury, making it the fourth leading cause of hospital admissions, economic analyses are justified.

TBI is a massive global problem causing death and disability to millions annually. Despite the social and financial ramifications of TBI, ${ }^{2}$ studies on the cost of TBI care are relatively few in number. The literature has revealed that cost estimates are widely variable, dependent upon severity, comorbidities, and a variety of socioeconomic issues, such as race, ethnicity, geography, income, and other markers of social status. Although this study represents an incremental step forward in our understanding of the burden of TBI, it is nonetheless an important one.

Malaysia is considered by the World Bank to be an upper middle-income country (MIC) at present, and economically stable MICs are considered to have positive effects on other parts of the world in terms of international financial stability. In-country health care expenditures, therefore, have ramifications not only for Malaysia, but if they are detrimental to the country's overall economy, they may have negative downstream consequences to other countries. It is also important for the government and the medical community to understand the impact of costs of care provided to its citizens, because much of that care is funded by the government (Ministry of Health). ${ }^{5}$

Furthermore, according to the World Bank, the nation has identified the poorest $40 \%$ of the population as an important segment on which to focus aid efforts, which have been provided (at least in part) in the form of direct material financial support to individuals. This group, like the lowest income segment of any population, is particularly vulnerable to unforeseen events causing economic hardship, such as an injury or illness requiring unexpected expenditures. Certainly, sustaining a TBI can financially challenge or even bankrupt an individual or a family, and for those already at financial risk, it can be ruinous. The financial obligation from the government may be additionally burdened by a large population of both survivors and family members who can no longer earn because they must forego gainful employment to care for the injured person. This vicious cycle can contribute to an ongoing public health burden in any country, and while beyond the scope of the You study, it will be important in the future to analyze downstream financial opportunity costs arising from TBI, in addition to the direct costs. TBI may have a disproportionate negative financial impact on people living in countries with lower incomes, and data are needed from a variety of environments to help clarify the mechanisms by which care is provided and ultimately target public health initiatives to reduce injury rates, decrease complication rates, and improve outcomes.

For example, in a truly integrated trauma and public health system, injury data should be collected and utilized to prioritize prevention efforts for the jurisdiction in question. ${ }^{3}$ The majority of severe TBI patients were motorcyclists in the study by You et al. ${ }^{5}$ The identification of motorized cycling as a major mechanism of injury can be used to advocate for effective expenditure of resources on preventive measures such as educational programs, safety initiatives, and even government actions aimed at reduction of motorcycle crashes in this region of Malaysia or indeed nationwide.

Having these data should inform efforts to improve the 
overall quality of care as well. For this center, targeting rate reductions for pneumonia and decubitus ulcers as the most common complications cited will not only improve clinical outcomes but will cost less, as pneumonia was an independent predictor of higher cost.

Finally, models for gathering data and performing economic analyses like the one done here will aid other countries. Particularly in those nations in which road traffic is increasing, people are susceptible to vehicle-related injury as traffic outpaces the growth of infrastructure, regulations, emergency services, and trauma systems. By collecting data on mechanism of injury, severity of injury, and the cost of care by age, procedure, complication rates, and the like, guidance can be provided to health authorities and medical providers. Health authorities can place resources where they are needed, and medical providers can focus clinical efforts on specific care improvements.

Although the World Health Organization (WHO) adopted standards for central nervous system injury surveillance in 1993, repositories of data regarding injury and both its direct and opportunity costs still contain limited information and have not adequately addressed the many confounders of ascribing true cost to a complex injury. It is critical, as registry science evolves and databases are deployed, to include at least some markers of cost of care. However, the vagaries of using billing code-based data are well known and little data exist on TBI coding accuracy. Studies do support the notion that International Classifications of Diseases (ICD) coding is insufficient for accurate characterization of epidemiological data in TBI. ${ }^{1,4}$ Numerous studies of other medical conditions have confirmed this finding. The authors rightly point out that the collection of flawed direct financial cost data may lead to "unfair comparisons and flawed policy choices." The use of strictly administrative data such as ICD codes, while informative, should not directly drive policy decisions, but should be taken together with other forms of data collection to help medical communities, health authorities, and payors make decisions about optimal care.

As You and colleagues have also pointed out, the true costs of care go far beyond those associated with hospitalization. While hospital costs can be gleaned more readily, the cost of rehabilitation, home modifications, home care, and follow-up for multiple aspects of recovery are typically difficult to capture. Furthermore, the opportunity costs of lost productivity not only by the patient but also family and friend caregivers is, while not completely intangible, not easy to estimate. Actuarial science can aid in the development of expected opportunity costs for given injuries, and other social sciences fields must be engaged to assist in these predictions.

This particular study only included individuals up to the age of 60 years. When considering the increasing older population worldwide, cost estimations must be adjusted to include lower opportunity costs for shorter life expectancies and earning potential of older individuals. The direct costs of care in older populations with comorbidities would, however, be anticipated to be higher. Indeed, in this particular study, the older the patient by increments of 1 year, the higher the cost of care. Population data collection via census mechanisms, together with injury rate data, can be added to data such as those presented here to predict overall cost of care anticipated from TBI for a given municipality, region, or country.

Perhaps most importantly, discussions of cost of care should not presume that patients do not benefit from treatment, nor should there be a presumption that they do not return to productivity. Indeed, aggressive management can lead to quite good functional outcomes and may be worth not only the financial costs to society, but all of the time, energy, and effort that goes into the complex management of TBI. We must not lose sight of our humanity in our decision-making or of the importance of aggressive medical care in rendering patients back to functionality. Early prognostication abilities for TBI in particular are limited, and the neurotrauma research community must continue to push for better prognostic tools and treatment methods for TBI. Certainly, caution must be employed in the interpretation of purely economic data not to advocate for a nihilistic approach to care, lest the practice of nonaggressive management lead to a self-fulfilling prophecy of poor outcomes.

In summary, efforts to assess the cost of care of TBI are challenged by incomplete or inaccurate databases, complexity of clinical care, lack of epidemiological data, and scanty use of actuarial cost prediction methodologies. This study represents an early attempt to document costs of care of TBI in one regional medical center in Malaysia, but will hopefully lead to other such analyses worldwide and redoubled efforts by public health bodies to focus on the epidemic of TBI-related morbidity and mortality by supporting data collection, appropriate emergency services, acute care, and after-care through functional survival and community reintegration.

https://thejns.org/doi/abs/10.3171/2018.2.FOCUS1888

\section{References}

1. Barker-Collo S, Theadom A, Jones K, Feigin VL, Kahan M: Accuracy of an International Classification of Diseases code surveillance system in the identification of traumatic brain injury. Neuroepidemiology 47:46-52, 2016

2. Dismuke CE, Walker RJ, Egede LE: Utilization and cost of health services in individuals with traumatic brain injury. Glob J Health Sci 7:156-169, 2015

3. Health Resources and Services Administration: Model Trauma System Planning and Evaluation. Washington, DC: U.S. Department of Health and Human Services, 2006 (https://www.hsdl.org/?view\&did=463554) [Accessed March 14, 2018]

4. St Germaine-Smith C, Metcalfe A, Pringsheim T, Roberts JI, Beck CA, Hemmelgarn BR, et al: Recommendations for optimal ICD codes to study neurologic conditions: a systematic review. Neurology 79:1049-1055, 2012

5. You X, Liew BS, Rosman AK, Musa KI, Idris Z: The estimated cost of surgically managed isolated traumatic head injury secondary to road traffic accidents. Neurosurg Focus 44(5):E7, 2018

\section{Disclosures}

The authors report no conflict of interest. 